\title{
A patient with cavernous sinus cavernoma who was difficult to diagnose by imaging modalities.
}

\author{
Akiko Marutani“, Kiyokazu Asada, Noriyuki Nishi, Tomonori Yamada \\ Department of Neurosurgery, National Hospital Organization, Osaka Minami Medical Center, 2-1 Kido-higashimachi, \\ Kawatinagano-shi, Osaka, Japan
}

\begin{abstract}
Cavernous Sinus Cavernous Hemangioma (CSCH) is a relatively rare vascular lesion, accounting for 0.4-2.0\% of tumors of the cavernous sinus. Due to multiple differential diagnoses, a diagnosis based on pre-operative imaging is very important. This case report describes a patient with $\mathrm{CSCH}$ in whom it was difficult to determine a pre-operative diagnosis based on imaging modalities.
\end{abstract}

Keywords: Cavernous sinus cavernoma, Cavernous sinus, Magnetic resonance imaging, Transnasal transsphenoidal surgery, Hematoma component.

Accepted on April 19, 2018

\section{Introduction}

This case is important because of the diagnosis change of the treatment varies depending on the type of tumor. Cavernous Sinus Cavernous Hemangioma $(\mathrm{CSCH})$ is a relatively rare vascular lesion; it only accounts for $0.4-2.0 \%$ of tumors of the cavernous sinus. Pre-operative imaging is very important for establishing a diagnosis as there are multiple differential diagnoses. This report describes a patient with $\mathrm{CSCH}$ who was difficult to diagnose pre-operatively by imaging modalities.

\section{Case Report}

\section{Patient}

The patient was a 49-year-old man. There was no relevant medical or family history.

The patient provided consent for the publication of this article.

The patient had undergone brain Magnetic Resonance Imaging (MRI) for vertigo at another hospital. He was referred to our department after the brain MRI findings had shown a tumor of the cavernous sinus. Neurologic examination showed no evidence of visual dysfunction or other symptoms of neurological deficits. No endocrine disorders were observed.

\section{Imaging findings}

Computed Tomography (CT) showed a well demarcated lowdensity area with a homogeneous interior in the upper region of the right sella turcica and encompassing the right (inferior) cavernous sinus. There were no obvious signs of calcification or bone destruction. MRI without Gadolinium (Gd) contrast enhancement showed a well-demarcated lesion with a diameter of $1.5 \mathrm{~cm}$ that appeared as a homogeneously low-intensity area on T1 and a homogeneously high-intensity area on T2 (Figure 1).

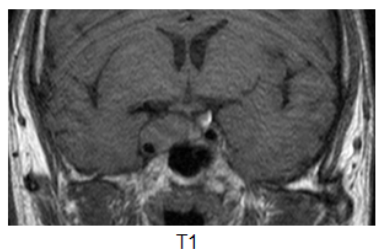

$\mathrm{R}$

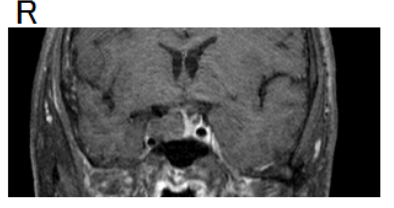

cor
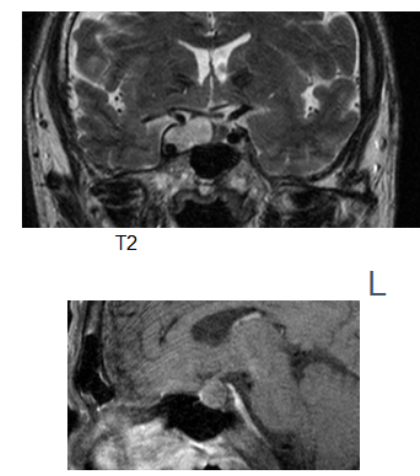

sag
Figure 1. Magnetic resonance imaging without Gadolinium contrast enhancement showed a well-demarcated lesion with a diameter of 1.5 $\mathrm{cm}$, appearing as a homogeneously low-intensity area on $T 1$ and a homogeneously high-intensity area on T2.

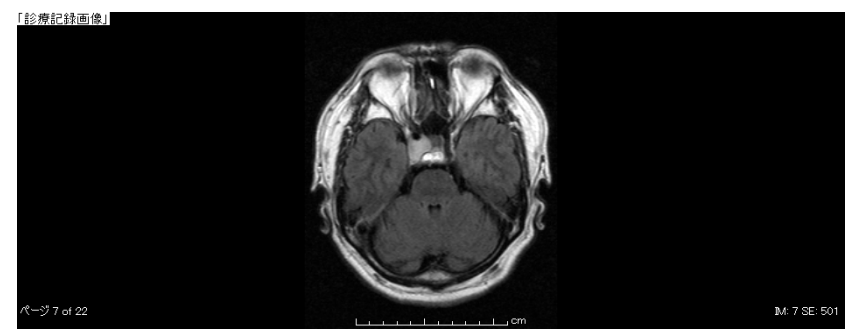

Figure 2. MRI also showed infiltration of the right internal carotid artery and around the right optic nerve with deviations to the right side; no deviations of the pituitary stalk were seen.

MRI also showed infiltration of the right internal carotid artery and around the right optic nerve with deviations to the right 
side, but no deviations of the pituitary stalk (Figure 2). Cerebral angiography showed no evidence of dense tumor staining.

\section{Treatment}

The cyst on the sella turcica and the pituitary adenoma were surgically removed through Transsphenoidal Surgery (TSS) for pituitary tumors.

An incision of the dura mater revealed that the tunica was coated with a hard, easily hemorrhaging red-colored tumor, which was excised surgically. Considerable bleeding was observed in the hard tissue; it was partially excised as it was difficult to excise the deeper regions of the lesion. The patient experienced no postoperative complications involving neurological deficits. Brain MRI performed 6 months after the surgery.

\section{Pathological findings}

Hematoxylin and eosin staining of the tumor tissue showed large and small vascular cavities that were separated from their original stratum of the vascular endothelium by a stroma of collagen fibers in the interstitial spaces. There were no interposing nerve tissues between the vessel lumens. The collagen fibers were positive in Elastica van Gieson staining, and immunostaining showed that the lesion was negative for EMA and vimentin and positive for FVIII. Based on these findings, the lesion was diagnosed as a cavernous sinus cavernoma.

\section{Discussion}

$\mathrm{CSCH}$ is a rare type of vascular tumor, accounting for approximately $3 \%$ of benign tumors of cavernous sinus origin. Symptoms may be neurological and include those involving the cranial nerves and presenting as diplopia and endocrine symptoms. Although surgery through the transsphenoidal bone is possible, complete removal of the lesion is difficult, and hemorrhaging is frequent [1]. Removal by craniotomy is complicated by the difficulty in controlling bleeding, with some patients experiencing serious sequelae and even death. In contrast, radiotherapy has been reported effective in the treatment of these tumors, with reductions in tumor size observed at irradiation levels of approximately $40 \mathrm{~Gy}$. Delayed effects of radiotherapy have also been reported [2].

High-field MRI T1 images of patients with CSCH show well demarcated lesions with low or constant intensity signals, whereas T2-weighted images show high-intensity signals, similar to those of cerebrospinal fluid [3]. On Gd-DiethyleneTriamine-Pentaacetic Acid (Gd-DTPA) contrast T1-weighted images, the enhancement effect often exhibits a high degree of homogeneity and gradual deep tumor staining. Different signal changes from brain parenchymal Cerebral Cavernous Malformation (CCM) have been observed, similar to those of internal deposits of hemorrhagic components including hemosiderin. Angiography has shown tumor blush in about $80 \%$ of patients with $\mathrm{CSCH}$. The lesions are fed by the internal carotid and middle cerebral arteries; in many patients, part or the entire vein is stained deeply in a spotted pattern.

Unlike intracranial cavernous angioma, cavernous sinus hemangioma is proliferative, with continuous malformations of blood vessels continuous with the vascular epithelium; some patients show thrombosis of the vascular lumen. The vascular lumen, localized to the cavernous sinus, is of a spongy or capillary nature; when it expands in size, it occupies the middle cranial fossa, despite remaining in the cavernous sinus at first. While often $\mathrm{CSCH}$ observed in middle-aged women, pregnancy in younger women may result in an increased size of the lesion [4].

Establishing a pre-operative differential diagnosis in this patient was difficult because of Gd contrast enhancement was not observed. $\mathrm{CSCH}$ differs from intracranial cavernoma, as the former shows no contrast effect. Moreover, blood flow may be deficient in $\mathrm{CSCH}$, resulting from the confined proliferation of intra-capsular thrombotic vascular cavities and hematoma components [4].

Because lesions with contrast effect display blood flow dynamics, cases in which the hematoma is the main component of the lesion are thought to result from deficient blood flow within the tumor or from ischemia of nutrient blood vessels secondary to hematomal ischemia. Signal changes may therefore be due to differences in the deposition of hematomal components such as hemosiderin.

The differential diagnosis of $\mathrm{CSCH}$ includes meningiomas, (trigeminal) schwannomas, malignant lymphomas, and metastatic brain tumors. Although meningiomas and $\mathrm{CSCH} /$ are morphologically similar, they can be differentiated by $\mathrm{T} 2$ weighted images high-intensity signals in meningiomas and low-density signals in $\mathrm{CSCH}$. Trigeminal schwannomas are characterized by non-uniform signal changes. CT-based characterization of the bone information of the skull base is useful for the diagnosis of chondromas and chordomas [5].

\section{Conclusion}

In this patient, establishing a pre-surgical differential diagnosis was difficult due to the lack of a hypercontrast effect with Gd. Internal deposition of different hematomal components such as hemosiderin may result in signal changes.

\section{References}

1. Lombardi D, Giovanelli M, de Tribolet N. Sellar and parasellar extra-axial cavernous hemangiomas. Acta Neurichir (Wien) 1994; 130: 47-54.

2. Sasaki T, Hayashi N, Tomura N, Tsuji E, Okada H, Kuwata T. A case of a cerebral cavernous malformation of the third ventricle that caused the syndrome of inappropriate secretion of antidiuretic hormone. Surg Neurol Int 2017; 8: 53.

3. Zhou LF, Mao Y, Chen L. Diagnosis and surgical treatment of cavernous sinus hemangiomas: an experience of 20 cases. Surg Neurol 2003; 60: 31-37. 
A patient with cavernous sinus cavernoma who was difficult to diagnose by imaging modalities

4. Shi J, Hang C, Pan Y, Liu C, Zhang Z. Cavernous hemangiomas in the cavernous sinus. Neurosurgery 1999; 45: $1308-1314$.

5. Toyoda K, Oba H. Diagnostic imaging of hemangiomas in the brain. Brain Nerve 2011; 63: 5-15 (in Japanese).
Department of Neurosurgery

National Hospital Organization

Osaka Minami Medical Center

Japan

\section{*Correspondence to}

Akiko Marutani 